\title{
Efficient Terahertz Generation Within InGaN/GaN Multiple Quantum Wells
}

\author{
Guan Sun, Guibao Xu, Yujie J. Ding, Senior Member, IEEE, Hongping Zhao, Guangyu Liu, Jing Zhang, \\ and Nelson Tansu, Senior Member, IEEE
}

\begin{abstract}
We have investigated terahertz (THz) generation from InGaN/GaN multiple quantum wells (QWs). For the laser pump power of $400 \mathrm{~mW}$ at $391 \mathrm{~nm}$, the highest THz output power is nearly $1 \mu \mathrm{W}$. Assuming that the output power quadratically scales up with the interaction length, such an output power corresponds to a normalized output power of $1.7 \mathrm{nW} / \mathrm{nm}^{2}$. The normalized output power measured on the InGaN/GaN multiple quantumwell structures correspond to probably one of the highest values ever reported among all different semiconductor and nonlinear materials. Following our measurements of the output spectrum, power, and polarization angle as functions of average pump intensity, incident angle, and pump polarization angle, respectively, we have attributed the mechanism for the $\mathrm{THz}$ generation from the InGaN/GaN QWs to the radiation of the dipoles, following the generation of the spatially separated electrons and holes under the strong built-in electric fields inherently present in the nitride-based quantum-well structures.
\end{abstract}

Index Terms-Broadband terahertz (THz) wave, built-in field, dipole radiation, InGaN/GaN quantum wells (QWs), spontaneous and piezoelectric polarizations.

\section{INTRODUCTION}

B ROADBANDterahertz (THz) pulses were generated primarily based on three mechanisms, i.e., optical rectification from second-order nonlinear materials [1], [2], photoconduction or photocurrent surge from electrooptic materials [3], and Cherenkov radiation from ferroelectric materials [4]. It was demonstrated that based on optical rectification in ZnTe [5] and $\mathrm{LiNbO}_{3}$ [6], high peak intensities on the order of $10 \mathrm{MW} / \mathrm{cm}^{2}$ can be reached. Such intensities are sufficiently high for investigating nonlinear fundamental phenomena in the $\mathrm{THz}$ region, such as harmonic generation [7], [8] and THz-enhanced UV emission from gas plasma [9]. It appears to us that semiconductor materials are more promising than ferroelectric materials, such as $\mathrm{LiNbO}_{3}$ for scaling up the output powers. This is due to the fact that semiconductor materials do not suffer from photorefractive damage. In addition, since index of refraction in $\mathrm{THz}$ is significantly lower for semiconductors, the $\mathrm{THz}$ output power can be inherently higher [10]. Moreover, broadband phase matching is feasible for certain materials, such as $\mathrm{ZnTe}, \mathrm{GaP}$,

Manuscript received February 13, 2010; revised March 11, 2010; accepted April 22, 2010. Date of publication June 21, 2010; date of current version February 4, 2011. This work was supported by the Defense Advanced Research Projects Agency through the U.S. Air Force Research Laboratory and the National Science Foundation under Award ECCS 0701421.

The authors are with the Department of Electrical and Computer Engineering and Center for Optical Technologies, Lehigh University, Bethlehem, PA 18015 USA (e-mail: gus208@lehigh.edu; gux206@lehigh.edu; yud2@lehigh.edu; hoz207@lehigh.edu; gul308@lehigh.edu; jiz209@lehigh.edu; tansu@lehigh. edu).

Color versions of one or more of the figures in this paper are available online at http://ieeexplore.iee.org.

Digital Object Identifier 10.1109/JSTQE.2010.2049343 and GaAs under the collinear configuration [10]. Besides optical rectification, photoconduction or photocurrent surge is another primary mechanism for $\mathrm{THz}$ generation from semiconductor materials [11]. When semiconductor quantum wells (QWs) were under a bias, the mechanism for $\mathrm{THz}$ generation was attributed to the creation of polarized electron-hole pairs [12]. However, in GaAs/AlGaAs QWs, an electric field must be externally applied. It was also demonstrated that $\mathrm{THz}$ pulses were generated due to the coherent oscillation of electrons in asymmetric-coupled QWs [13]. In nitride-based heterostructures, however, a strong built-in field originates from spontaneous and piezoelectric polarizations with its magnitude up to $3.1 \mathrm{MV} / \mathrm{cm}$ for InGaN/GaN QWs [14] and 9.2 MV/cm for GaN/AlN QWs [15]. The large polarization fields result in significant charge-separation effect in the nitride-based QWs (i.e., InGaN/GaN QWs). It is worth noting that by employing novel QW structures with improved electron-hole wavefunction overlaps, the charge-separation effect can be engineered [16]-[19]. In the past, THz emission from InGaN/GaN QWs was observed without the presence of an external electric field [20]. The mechanism for the THz generation was attributed to dynamical screening of a strong built-in electric field [21]. Such a built-in electric field is inherently present in the InGaN/GaN QWs grown on the [0 $\left.\begin{array}{llll}0 & 0 & 1 & 1\end{array}\right]$ direction [14], as mentioned earlier.

In this paper, we report our result following our investigation of efficient $\mathrm{THz}$ generation from InGaN/GaN QWs achieved at room temperature. We have directly measured the average $\mathrm{THz}$ output power to be as high as $0.96 \mu \mathrm{W}$, generated by eight periods of the $\mathrm{In}_{0.25} \mathrm{Ga}_{0.75} \mathrm{~N} / \mathrm{GaN}$ QWs. The characteristics measured on the InGaN/GaN QWs, such as THz output spectrum and power versus incident angle, THz output power and polarization versus pump polarization angle, and $\mathrm{THz}$ output power and polarization versus azimuth angle, are essentially the same as those due to the radiation originating from the instantaneous generation of dipoles (i.e., photocurrent surge). Within the range of the pump intensities used for investigating the $\mathrm{THz}$ generation, the energy for the band-to-band transition peak exhibits a small amount of blue shift as the pump intensity is increased. Following our analysis, we have concluded that the mechanism for $\mathrm{THz}$ generation is primarily the instantaneous generation of spatially separated electron-hole pairs resulting in dipole radiation. Our comparison with the results obtained on the InGaN thin film reveals that the output power from the InGaN/GaN multiple QWs is dramatically scaled up.

\section{InGaN/GaN QWs}

The growth of InGaN/GaN QWs structures was carried out on a $2.8-\mu \mathrm{m}$-thick unintentionally doped $\mathrm{GaN}$ (background electron density of $\sim 4 \times 10^{16} \mathrm{~cm}^{-3}$ ) template on a $c$-plane sapphire substrate by metal-organic chemical vapor deposition 
(MOCVD). The growth of the GaN template was performed at $1080^{\circ} \mathrm{C}$ by employing 35 -nm-thick low-temperature-grown $\left(T_{g}=525^{\circ} \mathrm{C}\right) \mathrm{GaN}$ buffer layer. Subsequently, eight periods of $\mathrm{In}_{0.25} \mathrm{Ga}_{0.75} \mathrm{~N} / \mathrm{GaN}$ QWs were deposited on the $\mathrm{GaN}$ template by MOCVD at $700^{\circ} \mathrm{C}$. The thicknesses of the $\mathrm{InGaN}$ well and GaN barrier layers are 3 and $12 \mathrm{~nm}$, respectively. In addition to the InGaN/GaN QWs, samples of $180 \mathrm{~nm} \mathrm{In}_{0.36} \mathrm{Ga}_{0.64} \mathrm{~N}$ and $2.8 \mu \mathrm{m} \mathrm{GaN} / \mathrm{sapphire}$ thin films were grown for making the comparison with the InGaN/GaN QWs.

\section{EXPERIMENTAL SETUP}

Broadband THz pulses were generated by a coherent radiation beam at the wavelength of $391 \mathrm{~nm}$ after frequency-doubling the output beam from a Ti:sapphire regenerative amplifier. Such an output beam can be used to generate electrons and holes in the conduction and valence bands of the $\mathrm{In}_{0.25} \mathrm{Ga}_{0.75} \mathrm{~N} / \mathrm{GaN} \mathrm{QWs}$, respectively. The pulse duration is $\sim 210 \mathrm{fs}$ and repetition rate is $250 \mathrm{kHz}$. The excitation beam was focused onto each sample by a positive lens with the focal length of $10 \mathrm{~cm}$. The THz radiation was collimated, and then, focused onto a bolometer or a pyroelectric detector by a pair of gold-coated parabolic mirrors. The first mirror was used to collimate the THz output beam, whereas the second one was used to focus it onto each detector. We measured the $\mathrm{THz}$ output beams in both the transmission and reflection geometries. In order to measure the energy of the band-to-band transition peak from the $\operatorname{In}_{0.25} \mathrm{Ga}_{0.75} \mathrm{~N} / \mathrm{GaN}$ QWs, we used the output beam from the same amplifier to generate the luminescence signal.

\section{THZ GENERATION}

In this section, we present our result on the $\mathrm{THz}$ generation from the $\operatorname{In}_{0.25} \mathrm{Ga}_{0.75} \mathrm{~N} / \mathrm{GaN}$ QWs, as well as the comparison studies with the InGaN and $\mathrm{GaN}$ thin-film samples.

Based on our measurements of the output powers from the $\mathrm{In}_{0.25} \mathrm{Ga}_{0.75} \mathrm{~N} / \mathrm{GaN} \mathrm{QWs}$, the highest output power is $0.956 \mu \mathrm{W}$ for an average pump power of $400 \mathrm{~mW}$ at $391 \mathrm{~nm}$. The densities of the photogenerated carriers following the absorption of the pump pulses are expected to increase linearly with the period of the QWs. By assuming that the output power quadratically increases with increasing the carrier densities, we have estimated the output power from a single period of the QW with the well thickness of $3 \mathrm{~nm}$ to be about $120 \mathrm{nW}$. Since the noise level of our pyroelectric detector operating at room temperature is $230 \mathrm{pW}$, such an output power provides us with a dynamic range as high as 520 . We can also determine the output power normalized by the square of the effective interaction length (i.e., $576 \mathrm{~nm}^{2}$ ) to be $1.66 \mathrm{nW} / \mathrm{nm}^{2}$. We believe that the normalized output power measured on the InGaN/GaN multiple QWs is probably one of the highest values ever reported among all different materials. For comparison, let us consider a GaSe crystal. According to our recent result [22], the average output power generated was $5.4 \mu \mathrm{W}$ for a GaSe thickness of $145 \mu \mathrm{m}$. Such an output power corresponds to the normalized output power of $2.6 \times 10^{-7} \mathrm{nW} / \mathrm{nm}^{2}$ (i.e., the output power normalized by the square of the thickness). Therefore, the output power normalized by the square of the effective interaction length, obtained by us on the InGaN/GaN multiple QWs, is seven orders of magnitude higher than that from the GaSe crystal. On the other hand, the average output power of $0.956 \mu \mathrm{W}$ is a factor of about 1900

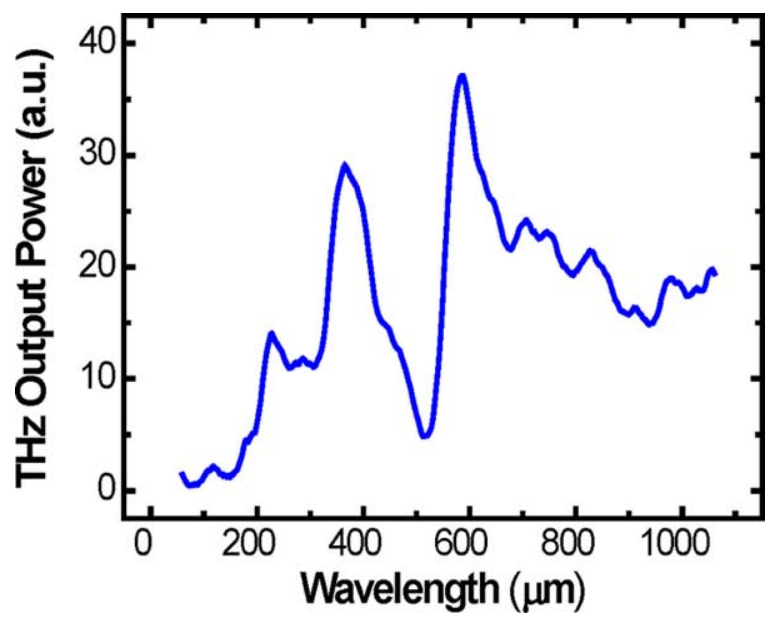

Fig. 1. Typical spectrum of THz wave emitted by $\mathrm{In}_{0.25} \mathrm{Ga}_{0.75} \mathrm{~N} / \mathrm{GaN}$ QWs The huge dip at $520 \mu \mathrm{m}$ and other modulations were caused by water-vapor absorption.

(i.e., three orders of magnitude) higher than that measured by us on a 25-nm-thick InN film sample [23].

In comparison, the output power from the 180-nm $\mathrm{In}_{0.36} \mathrm{Ga}_{0.64} \mathrm{~N}$ thin-film sample was measured to be $112 \mathrm{nW}$. Assuming the quadratic dependence of the output power on the thickness, the output power from a $3-\mathrm{nm} \mathrm{In}_{0.36} \mathrm{Ga}_{0.64} \mathrm{~N}$ film only is expected to be $31.1 \mathrm{pW}$, which is a factor of about 3800 lower than that deduced from a single InGaN/GaN QW. On the other hand, for the $2.8-\mu \mathrm{m} \mathrm{GaN}$ film, the THz signal was buried by the noise of the bolometer. Such comparisons among the output powers generated from three samples reveal that the mechanism for the $\mathrm{THz}$ generation from the InGaN/GaN QWs must be fundamentally different from that for the InGaN thin-film sample.

We have measured the spectrum of the $\mathrm{THz}$ output beam generated by the $\operatorname{In}_{0.25} \mathrm{Ga}_{0.75} \mathrm{~N} / \mathrm{GaN}$ QWs by directly studying the diffraction of the $\mathrm{THz}$ beam by a set of the rotating gratings. Based on Fig. 1, we can see that the frequencies of the $\mathrm{THz}$ output roughly span the frequency range from $300 \mathrm{GHz}$ to $1.5 \mathrm{THz}$. The huge dip located at $520 \mu \mathrm{m}(577 \mathrm{GHz})$ is caused by water-vapor absorption. Based on our measurements of the $\mathrm{THz}$ output spectra at different pump intensities, the spectral features illustrated in Fig. 1 are independent of the pump intensities. Therefore, the dynamical screening of the built-in electric field in our QWs is negligible, unlike the results obtained in [20] and [21]. Such a conclusion is supported by the measurement of the dependence of the $\mathrm{THz}$ output power on the pump intensity, as shown by Fig. 2. One can see from Fig. 2 that for the pump intensities below $13.6 \mathrm{~W} / \mathrm{cm}^{2}$, the power dependence exhibited a nearly square law. Therefore, within such a range of the pump intensities, we are certain that the dynamical screening of the built-in field is insignificant.

Above $13.6 \mathrm{~W} / \mathrm{cm}^{2}$, the power dependence significantly deviates from the quadratic dependence. We can rule out the screening of the built-in electric field [20], [24], [25], similar to screening the surface field in InP observed in the past [11], as a possible mechanism for causing such a deviation. Indeed, according to our measurement of the energy for the bandto-band transition peak in the QWs, the shift of the peak is roughly $6 \mathrm{meV}$, as the pump intensity is increased from 1.5 to $30 \mathrm{~W} / \mathrm{cm}^{2}$, as illustrated by Fig. 3. Since the linewidth of the photoluminescence peak is about $100 \mathrm{meV}$, such a small amount 


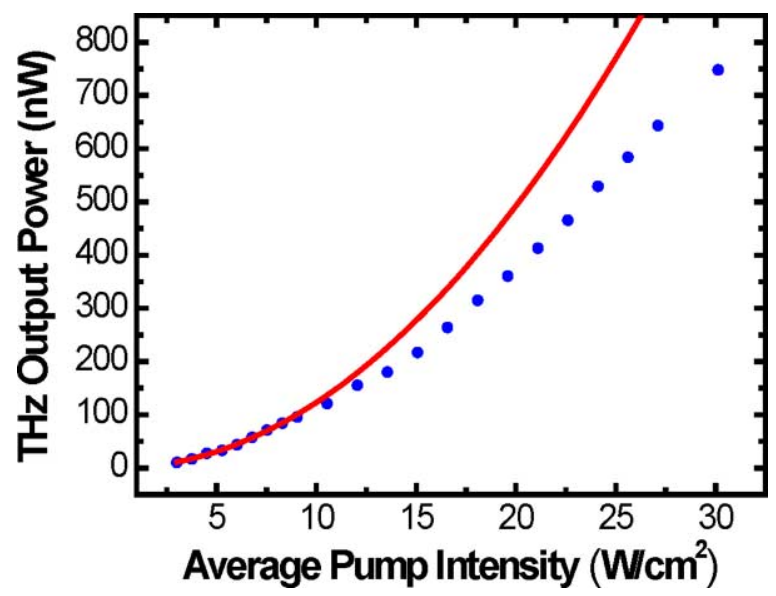

Fig. 2. Average THz output power generated from $\operatorname{In}_{0.25} \mathrm{Ga}_{0.75} \mathrm{~N} / \mathrm{GaN}$ multiple QWs was measured as a function of average pump intensity. Dots correspond to our data. Solid curve correspond to quadratic fit to the first nine pump powers from the low-intensity end.

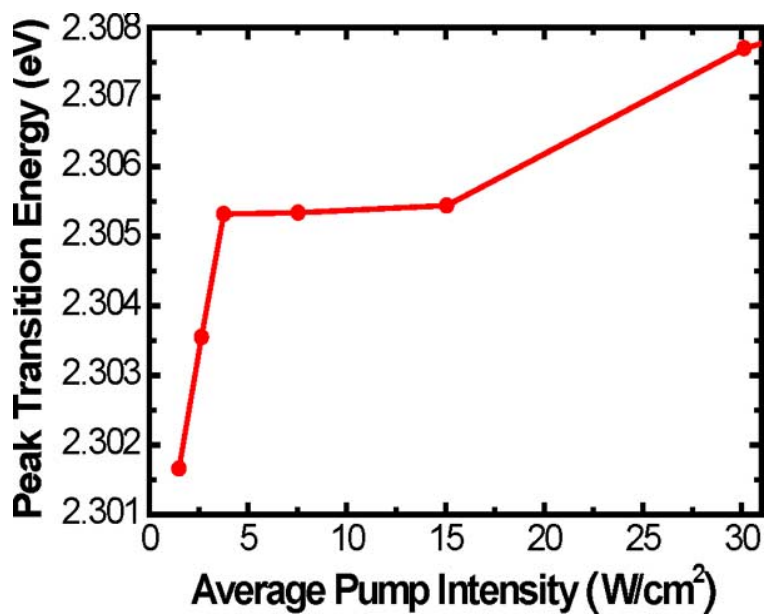

Fig. 3. Transition energy of $\operatorname{In}_{0.25} \mathrm{Ga}_{0.75} \mathrm{~N} / \mathrm{GaN} \mathrm{QW}$ s as a function of average pump intensity at room temperature.

of the shift determined from the photoluminescence peaks is only $6 \%$ of the photoluminescence linewidth, and therefore, it may be subject to a large error. Moreover, compared with the previous result [21], [24], the amount of the blue shift deduced by us is much lower. As we increased the pump intensity above $30 \mathrm{~W} / \mathrm{cm}^{2}$, the photoluminescence peak was further blue-shifted. However, the THz output power was significantly reduced. Such a trend is completely different from that observed in [20]. Therefore, the dynamical screening of the built-in field did not play a significant role within the pump intensities of $13.6-30 \mathrm{~W} / \mathrm{cm}^{2}$. A plausible mechanism causing the deviation observed in Fig. 2 can be the increase in the absorption of the $\mathrm{THz}$ wave by the photogenerated carriers, which was previously evidenced and investigated for pulsed-MOCVD-grown InN alloy [25]-[27].

In order to further understand the mechanism for the $\mathrm{THz}$ generation in our InGaN/GaN QWs, we first measured the output power of the $\mathrm{THz}$ beam propagating in the transmission direction as a function of the incident angle for the pump beam, as shown in Fig. 4. According to Fig. 4, when the incident angle for the pump beam was zero, the $\mathrm{THz}$ output power was close to zero. The highest $\mathrm{THz}$ output power occurred at an

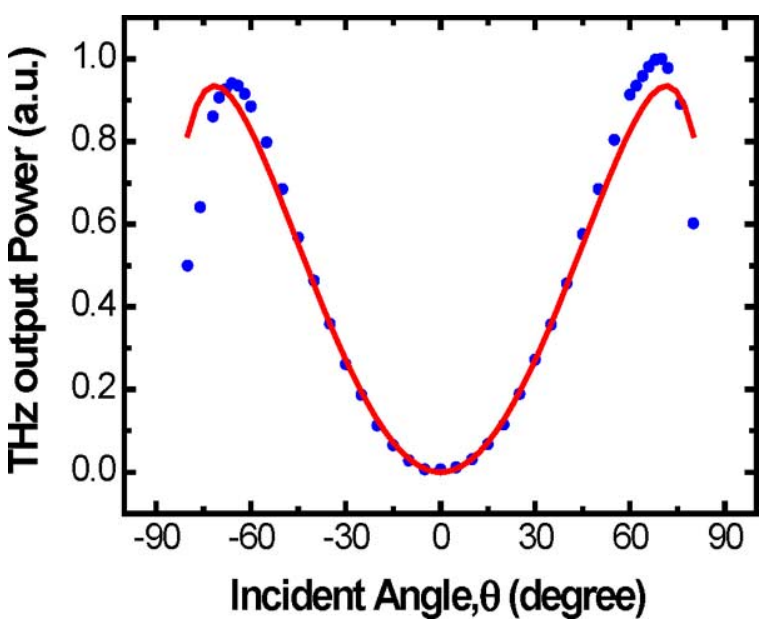

Fig. 4. THz output power generated by $\mathrm{In}_{0.25} \mathrm{Ga}_{0.75} \mathrm{~N} / \mathrm{GaN}$ multiple QWs was measured as a function of incident angle defined as the angle of surface normal being formed with the pump beam. Dots correspond to data points. Solid curve correspond to data fitting using (1). During our measurement, the pump beam was always $p$-polarized.

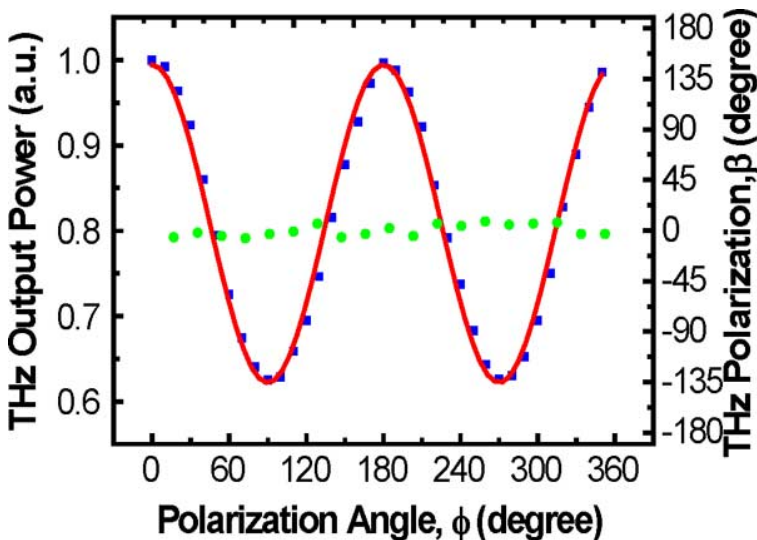

Fig. 5. Polarization dependences of $\mathrm{THz}$ output power generated by $\mathrm{In}_{0.25} \mathrm{Ga}_{0.75} \mathrm{~N} / \mathrm{GaN}$ multiple $\mathrm{QW}$ s and $\mathrm{THz}$ polarization angle. Polarization angle of pump beam is defined as the angle between the pump polarization and the incident plane. Polarization angle of $\mathrm{THz}$ wave, defined as the angle between the $\mathrm{THz}$ polarization and the incident plane, was determined by wire-grid polarizer. Squares designate average output power as function of pump polarization. Dots correspond to polarization angle of $\mathrm{THz}$ beam measured versus pump polarization. Solid curve is a theoretical result after taking into consideration Fresnel reflection.

incident angle of $72^{\circ}$, which is close to the Brewster angle for the pump beam. After taking into consideration the Fresnel reflection for the pump beam, the $\mathrm{THz}$ output power $\left(P_{\mathrm{THz}}\right)$ can be approximated by the following expression:

$$
P_{\mathrm{THz}} \approx f(\theta) \sin ^{2}(\theta)
$$

where $f(\theta)$ describes the contribution originating from the Fresnel reflection of the pump beam and $\sin ^{2}(\theta)$ represents a typical angle distribution of the dipole radiation. Our data can be well fitted by using (1), which is illustrated by Fig. 4. Thus, we conclude that the angular distribution of the THz radiation is consistent with that of the $\mathrm{THz}$ generation due to the dipole radiation.

Besides the angular distribution of the $\mathrm{THz}$ radiation, we also measured the dependences of the $\mathrm{THz}$ output power and polarization on the polarization of the pump beam and azimuth angle. Fig. 5 illustrates our result following the measurement of the $\mathrm{THz}$ output power as a function of the pump polarization 


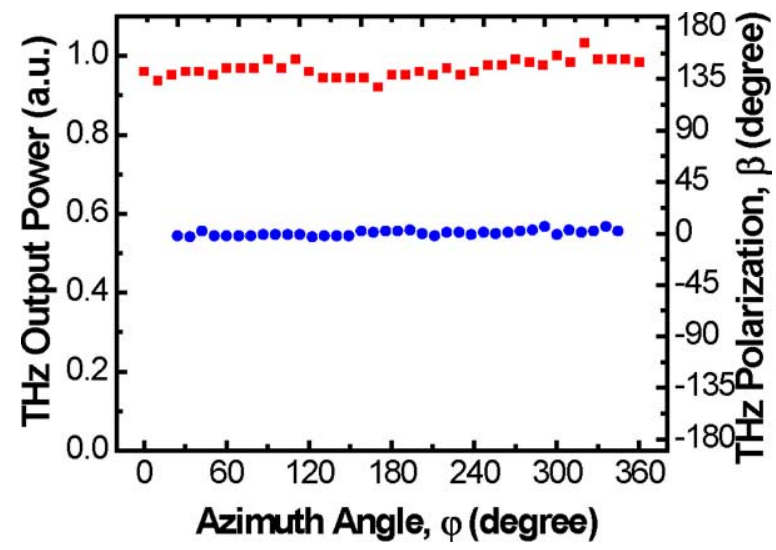

Fig. 6. Dependences of $\mathrm{THz}$ output power and $\mathrm{THz}$ polarization on azimuth angle for $\mathrm{In}_{0.25} \mathrm{Ga}_{0.75} \mathrm{~N} / \mathrm{GaN}$ multiple QWs. Azimuth angle is defined as the angle between the projection of the pump polarization onto the sample surface and a direction within the sample surface. Squares correspond to average output powers whereas dots represent polarization angles of $\mathrm{THz}$ beam.

angle in the reflection geometry. In such a case, we set the incident angle to be around $72^{\circ}$ in order to collect the maximum amount of the $\mathrm{THz}$ output power. One can see from Fig. 5 that the $\mathrm{THz}$ output power periodically oscillates as a function of the pump polarization angle. After taking into consideration the Fresnel reflection of the pump beam, our data can be well fitted by our theoretical curve, as shown by Fig. 5. This implies that the oscillation of the THz output power as a function of the pump polarization angle is primarily caused by the dependence of the Fresnel refraction on the polarization angle of the pump beam. Therefore, the dependence of the THz output power on the pump polarization angle is consistent with that of the $\mathrm{THz}$ generation due to the dipole radiation. Furthermore, one can see from Fig. 5 that the $\mathrm{THz}$ polarization angle is always around zero, i.e., the $\mathrm{THz}$ output beam was p-polarized with its polarization lying in the incidence plane. This is again consistent with our claim that the mechanism of $\mathrm{THz}$ generation is the dipole radiation.

We measured the dependences of the $\mathrm{THz}$ output power and polarization angle on the azimuth angle from the $\operatorname{In}_{0.25} \mathrm{Ga}_{0.75} \mathrm{~N} /$ GaN QWs in the reflection geometry. During these measurements, we set the incident and polarization angles of the pump beam to $35^{\circ}$ and $0^{\circ}$, respectively. According to Fig. 6 , we can see that both the THz output power and the polarization angle do not change when increasing the azimuth angle. Such results are also consistent with the $\mathrm{THz}$ generation due to the dipole radiation. Indeed, different azimuth angles would not affect the densities of the photogenerated electrons and holes, and therefore, they would not affect the $\mathrm{THz}$ output power and polarization.

After analyzing the data obtained from our measurements, we conclude that the mechanism for the $\mathrm{THz}$ generation from the InGaN/GaN QWs is the radiation of the dipoles, following the creation of the spatially separated electrons and holes. Such a mechanism was used to explain the $\mathrm{THz}$ radiation from GaAs/AlGaAs QWs under an external field [12]. According to [12], the spectrum of the $\mathrm{THz}$ radiation was more or less independent of the external electric field. Therefore, the $\mathrm{THz}$ spectrum was primarily determined by the frequency spectrum of the pump beam. On the other hand, according to [10], the low and high frequencies defining the bandwidth of the THz output are inversely proportional to the pulsewidth of the pump laser. Since the pulsewidth for the laser beam used in [12] was $100 \mathrm{fs,}$ the high-frequency components extended well beyond $2.5 \mathrm{THz}$. In our case, however, since we set the pulsewidth to $210 \mathrm{fs}$, the high-frequency components were expected to extend well beyond $1.2 \mathrm{THz}$. According to Fig. 1, this frequency is close to the value estimated from the spectrum. However, according to [21], the spectrum of the THz output was expected to strongly depend on the pump intensity due to dynamical screening of the built-in fields. Such a dependence is absent from our experiment. Moreover, according to [12], the electron-hole dipole moment is proportional to the built-in electric field. Therefore, due to the presence of the extremely high built-in electric fields in our InGaN/GaN QWs originating from the spontaneous and piezoelectric polarizations, we generated sufficiently high output powers. On the other hand, for the 180-nm-thick InGaN thin-film sample, photocurrent surge due to the diffusion and drift of the photogenerated carriers is the dominant mechanism for the THz generation. In such a case, however, the surface field is much lower than the built-in fields inherently present in the InGaN/GaN QWs. Such a huge difference in electric fields is the main reason for a dramatically reduced output power achieved from the 180-nm-thick InGaN thin-film sample.

\section{CONCLUSION}

In conclusion, we have efficiently generated broadband $\mathrm{THz}$ signals from InGaN/GaN multiple QWs. The THz emission from such a structure is attributed to the radiation of dipoles consisting of the photogenerated and spatially separated electrons and holes upon the illumination of the pump pulses at $391 \mathrm{~nm}$. Considering the fact that the total thickness of the eight periods of the InGaN/GaN QWs is about $24 \mathrm{~nm}$, the measured output power normalized by the square of the total thickness reaches $1.7 \mathrm{nW} / \mathrm{nm}^{2}$, which is probably one of the highest values ever reported. Such a high normalized output power was achieved by utilizing the very high built-in electric fields inherently present in the QWs to spatially separate electrons and holes in order to instantaneously create large dipoles. The proposed mechanism for the $\mathrm{THz}$ generation, i.e., the radiation of the dipoles due to the instantaneous generation of spatially separated electrons and holes, is consistent with our measurements of the spectrum of the THz output as well as the dependences of the THz output power and polarization angle on pump intensity, incident angle, polarization angle of the pump beam, and azimuth angle.

It was predicted in the past that wurtzite GaN/AlGaN QWs possess very high second-order nonlinear coefficients enhanced by strong built-in fields [28]. We believe that these second-order nonlinearities may play an important role under the pump intensities much higher than those used under our investigation. At such a pumping condition, it may be feasible for us to use InGaN/GaN QWs to detect THz waves based on frequency upconversion in second-order nonlinear materials [29], [30], as previously demonstrated. According to [22] and [23], these secondorder nonlinear coefficients can be dramatically enhanced under the condition of the resonant excitation.

The unique aspect of nitride-based semiconductor heterostructures, such as QWs offers an opportunity for us to engineer the structure of the QWs in order to further increase the $\mathrm{THz}$ output power. For example, for the InGaN/GaN QWs, the THz output power may be further increased by optimizing the widths of the wells and barriers, or by employing novel nitride-based QW structures [16]-[19], [31]. In addition, according to [10], 
the average output power can be increased to about $40 \mu \mathrm{W}$, i.e., by a factor of 40 , by reducing the pulsewidth of the pump beam to $60 \mathrm{fs}$ from $210 \mathrm{fs}$. In the future, we are going to investigate how the $\mathrm{THz}$ output power will scale with the temperature and external electric field.

\section{ACKNOWLEDGMENT}

The authors would like to thank $\mathrm{X}$. Mu for making earlier measurements on the structure studied here.

\section{REFERENCES}

[1] K. H. Yang, P. L. Richards, and Y. R. Shen, "Generation of far-infrared radiation by picosecond light pulses in LiNbO3," Appl. Phys. Lett., vol. 19, no. 9, pp. 320-323, Nov. 1971.

[2] L. Xu, X.-C. Zhang, and D. H. Auston, "Terahertz beam generation by femtosecond optical pulses in electro-optic materials," Appl. Phys. Lett., vol. 61 , no. 15 , pp. $1784-1786,1992$.

[3] D. H. Auston, K. P. Cheung, and P. R. Smith, "Picosecond photoconducting Hertzian dipoles," Appl. Phys. Lett., vol. 45, no. 3, pp. 284-286, Aug. 1984.

[4] D. H. Auston, K. P. Cheung, J. A. Valdmanis, and D. A. Kleinman, "Cherenkov radiation from femtosecond optical pulses in electro-optic media," Phys. Rev. Lett., vol. 53, no. 16, pp. 1555-1558, Oct. 1984.

[5] F. Blanchard, L. Razzari, H. C. Bandulet, G. Sharma, R. Morandotti, J. C. Kieffer, T. Ozaki, M. Reid, H. F. Tiedje, H. K. Haugen, and F A. Hegmann, "Generation of $1.5 \mu \mathrm{J}$ single-cycle terahertz pulses by optical rectifications from a large aperture ZnTe crystal," Opt. Exp., vol. 15 no. 20, pp. 13212-13220, Sep. 2007.

[6] K.-L. Yeh, M. C. Hoffmann, J. Hebling, and K. A. Nelson, "Generation of $10 \mu \mathrm{J}$ ultrashort terahertz pulses by optical rectification," Appl. Phys. Lett., vol. 90, pp. 171121-1-171121-3, Apr. 2007.

[7] Y. J. Ding, "Terahertz parametric converters by use of novel metallicdielectric hybrid waveguides," J. Opt. Soc. Amer. B, vol. 23, no. 7 , pp. 1354-1359, Jul. 2006

[8] J. Hebling, K.-L. Yeh, M. C. Hoffmann, and K. A. Nelson, "High-power THz generation, THz nonlinear optics, and THz nonlinear spectroscopy," IEEE J. Sel. Topics Quantum Electron., vol. 14, no. 2, pp. 345-353, Apr. 2008.

[9] J. Liu and X.-C. Zhang, "Terahertz-radiation-enhanced emission of fluorescence from gas plasma," Phys. Rev. Lett., vol. 103, pp. 235002-1235002-4, Dec. 2009.

[10] Y. J. Ding, "Efficient generation of high-power quasi-single-cycle $\mathrm{THz}$ pulses from single infrared beam in second-order nonlinear medium," Opt. Lett., vol. 29, pp. 2650-2652, Nov. 2004.

[11] B. I. Greene, J. F. Federici, D. R. Dykaar, A. F. J. Levi, and L. Pfeiffer, "Picosecond pump and probe spectroscopy utilizing freely propagating terahertz radiation," Opt. Lett., vol. 16, no. 1, pp. 48-49, Jan. 1991.

[12] P. C. M. Planken, M. C. Nuss, W. H. Knox, D. A. B. Miller, and K. W. Goossen, "THz pulses from the creation of polarized electron-hole pairs in biased quantum wells," Appl. Phys. Lett., vol. 61, pp. 2009-2011, Oct. 1992.

[13] H. G. Roskos, M. C. Nuss, J. Shah, K. Leo, D. A. B. Miller, A. M. Fox, S. Schmitt-Rink, and K. Köhler, "Coherent submillimeter-wave emission from charge oscillations in a double-well potential," Phys. Rev. Lett., vol. 68, pp. 2216-2219, Apr. 1992.

[14] A. Hangleiter, F. Hitzel, S. Lahmann, and U. Rossow, "Composition dependence of polarization fields in GaInN/GaN quantum wells," Appl. Phys. Lett., vol. 83, no. 6, pp. 1169-1171, Aug. 2003

[15] C. Adelmann, E. Sarigiannidou, D. Jalabert, Y. Hori, J.-L. Rouvière, B. Daudin, S. Fanget, C. Bru-Chevallier, T. Shibata, and M. Tanaka, "Growth and optical properties of GaN/AlN quantum wells," Appl. Phys. Lett., vol. 82, no. 23, pp. 4154-4156, Jun. 2003.

[16] R. A. Arif, Y. K. Ee, and N. Tansu, "Polarization engineering via staggered InGaN quantum wells for radiative efficiency enhancement of light emitting diodes," Appl. Phys. Lett., vol. 91, no. 9, pp. 091110-1-091110-3, Aug. 2007.

[17] R. A. Arif, H. P. Zhao, Y. K. Ee, and N. Tansu, "Spontaneous emission and characteristics of InGaN quantum wells light emitting diodes," IEEE J. Quantum Electron., vol. 44, no. 5/6, pp. 573-580, May/Jun. 2008.

[18] H. Zhao, R. A. Arif, and N. Tansu, "Design analysis of staggered InGaN quantum wells light-emitting diodes at 500-540 nm," IEEE J. Sel. Topics Quantum Electron., vol. 15, no. 4, pp. 1104-1114, Jul./Aug. 2009.
[19] H. Zhao, G. S. Huang, G. Liu, X. H. Li, J. D. Poplawsky, S. T. Penn, V. Dierolf, and N. Tansu, "Growths of staggered InGaN quantum wells light-emitting diodes emitting at 520-525 nm employing graded growthtemperature profile," Appl. Phys. Lett., vol. 95, no. 6, pp. 061104-1061104-3, Aug. 2009.

[20] D. Turchinovich, P. Uhd Jepsen, B. S. Monozon, M. Koch, S. Lahmann, U. Rossow, and A. Hangleiter, "Ultrafast polarization dynamics in biased quantum wells under strong femtosecond optical excitation," Phys. Rev. B., vol. 68, no. 24, pp. 241307-1-241307-4, Dec. 2003.

[21] D. Turchinovich, B. S. Monozon, and P. Uhd Jepsen, "Role of dynamical screening in excitation kinetics of biased quantum wells: Nonlinear absorption and ultrabroadband terahertz emission," J. Appl. Phys., vol. 99, no. 1, pp. 013510-1-013510-8, Jan. 2006.

[22] X. Mu, I. B. Zotova, and Y. J. Ding, "Power scaling on efficient generation of ultrafast terahertz pulses," IEEE J. Sel. Topics Quantum Electron., vol. 14, no. 2, pp. 315-332, Apr. 2008

[23] X. Mu, Y. J. Ding, K. Wang, D. Jena, and Y. B. Zotova, "Resonant terahertz generation from InN thin films," Opt. Lett., vol. 32, no. 11, Jun. 2007.

[24] Y. M. Park, J. K. Son, H. J. Chung, C. Sone, and Y. Park, "InGaN multiquantum well structure with a reduced internal electric field and carrier decay process by tunneling," Appl. Phys. Lett., vol. 95, no. 23, pp. 231917 1-231917-3, Dec. 2009.

[25] H. Zhao, R. A. Arif, Y. K. Ee, and N. Tansu, "Self-consistent analysis of strain-compensated InGaN-AlGaN quantum wells for lasers and light emitting diodes," IEEE J. Quantum Electron., vol. 45, no. 1-2, pp. 66-78, Jan./Feb. 2009

[26] G. Xu, Y. J. Ding, H. Zhao, G. Liu, M. Jamil, N. Tansu, I. B. Zotova, C. E. Stutz, D. E. Diggs, N. Fernelius, F. K. Hopkins, C. S. Gallinat, G. Koblmüller, and J. S. Speck, "THz generation from InN films due to destructive interference between optical rectification and photocurrent surge," Semicond. Sci. Technol., vol. 25, no. 1, pp. 015004-1-015004-5, Jan. 2010.

[27] M. Jamil, H. P. Zhao, J. Higgins, and N. Tansu, "MOVPE and photoluminescence of narrow band gap $(0.77 \mathrm{eV}) \mathrm{InN}$ on GaN/sapphire by pulsed growth mode," Phys. Stat. Sol. A, vol. 205, no. 12, pp. 2886-2891, Dec. 2008

[28] A. Liu, S.-L. Chuang, and C. Z. Ning, "Piezoelectric field-enhanced second-order nonlinear optical susceptibilities in wurtzite GaN/AlGaN quantum wells," Appl. Phys. Lett., vol. 76, no. 3, pp. 333-335, Jan. 2000.

[29] W. Shi, Y. J. Ding, N. Fernelius, and F. K. Hopkins, "Observation of difference-frequency generation by mixing of terahertz and near-infrared laser beams in a GaSe crystal," Appl. Phys. Lett., vol. 88, no. 10, pp. 101101-1-101101-3, Mar. 2006.

[30] M. J. Khan, J. C. Chen, and S. Kaushik, "Optical detection of terahertz radiation by using nonlinear parametric upconversion," Opt. Lett., vol. 32, no. 12, pp. 3248-3250, Nov. 2007.

[31] H. Zhao, R. A. Arif, and N. Tansu, "Self consistent gain analysis of type-II 'W' InGaN-GaNAs quantum well lasers," J. Appl. Phys., vol. 104, no. 4, pp. 043104-1-043104-7, Aug. 2008.

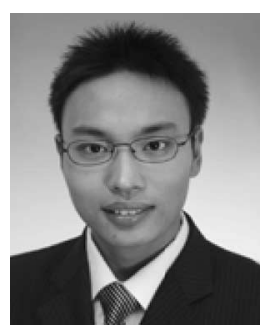

Guan Sun received the B.S. degree from Fudan University, Shanghai, China, in 2008. He is currently working toward the Ph.D. degree in the Department of Electrical and Computer Engineering, Lehigh University, Bethlehem, PA.

His current research interests include terahertz generation, applications, and III-nitride-based lightemitting diodes.

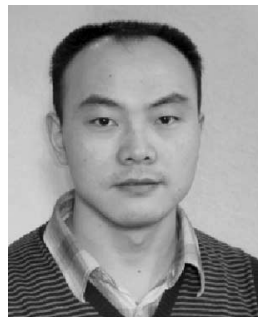

Guibao Xu received the B.E. and Ph.D. degrees from Shandong University, Jinan, China, in 2000 and 2006, respectively.

From 2004 to 2006, he was a Visiting Scholar at Max-Born-Institut, Berlin, Germany, and Institut National de Métrologie, Paris, France. Since 2006, he has been a Postdoctoral Fellow and a Research Associate in the Department of Electrical and Computer Engineering, Lehigh University, Bethlehem, PA. He is the author or coauthor of more than 40 refereed journal articles in nonlinear optical effects and devices, two-photon upconversion organic compounds, solid-state lasers, Raman scattering in nitride heterostructure, and terahertz $(\mathrm{THz})$ generation. His current research interests include $\mathrm{THz}$ generation, amplification, detection and application, and nonlinear optical devices. 


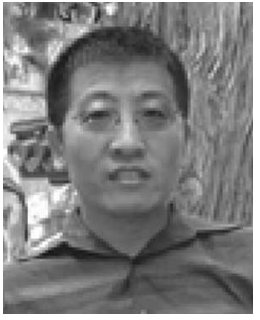

Yujie J. Ding (M'04-SM'05) received the B.S. degree from Jilin University, Jilin, China, in 1984, the M.S.E.E. degree from Purdue University, West Lafayette, IN, in 1987, and the Ph.D. degree from The Johns Hopkins University, Baltimore, MD, in 1990.

From 1990 to 1992, he was a Postdoctoral Fellow, and then, an Associate Research Scientist at The Johns Hopkins University. From 1992 to 1999, he was an Assistant and an Associate Professor of physics at Bowling Green State University, Bowling Green, OH. From 1999 to 2002, he was an Associate Professor of physics at the University of Arkansas, Fayetteville. In 2002, he joined Lehigh University, Bethlehem, PA, where he is currently a Professor in the Department of Electrical and Computer Engineering. He is the author or coauthor of more than 140 refereed journal articles in optoelectronics, nonlinear optics, and quantum electronics. His research interests include terahertz generation, amplification and detection, nanostructures and nanodevices, Raman scattering in nitride heterostructures, and their applications.

Prof. Ding is a Fellow of the Optical Society of America. He is the recipient of the Class of 1961 Professorship from Lehigh University in 2003 and the Outstanding Young Scholar Award from Bowling Green State University in 1996.

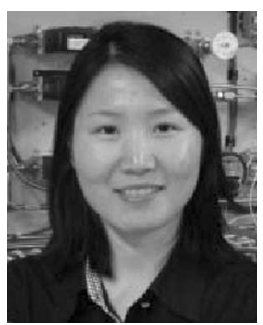

Hongping Zhao received the B.S. degree in physics from Nanjing Normal University, Nanjing, China, in 2003, the M.S. degree in electrical engineering from Southeast University, Nanjing, in 2006. Since January 2007, she has been working toward the Ph.D. degree in the Department of Electrical and Computer Engineering, Lehigh University, Bethlehem, PA.

Her current research interests include device physics, epitaxial growth, fabrication of semiconductor optoelectronics devices based on III-nitride semiconductor nanostructures, fundamental studies and approaches to improve internal quantum efficiency of visible III-nitride-based light emitting diodes especially emitting in the green regime, investigation of novel quantum well designs to enhance optical gain for nitride-based lasers for high-performance lasers, and metal-organic chemical vapor deposition epitaxy of InN material for quantum dots and solar cell applications. She has authored or coauthored more than 55 refereed journal and conference publications.

Ms. Zhao was the recipient of the 2008 and 2009 SPIE Educational Scholarship in optical science and engineering, and the Sherman Fairchild Fellowship on solid-state studies.

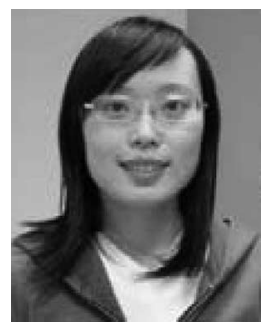

Guangyu Liu received the B.S. degree in electronic science and technology from the Huazhong University of Science and Technology, Wuhan, China, in 2008. Since July 2008, she has been working toward the Ph.D. degree in the Department of Electrical and Computer Engineering and the Center for Optical Technologies, Lehigh University, Bethlehem, PA.

Her current research interests include III-nitride semiconductor nanostructures and optoelectronics devices, covering the theoretical/computational, metal-organic chemical vapor deposition (MOCVD) growth, device fabrication technology, the pursuit of high-performance III-nitride quantum cascade lasers designs, device physics and growths of III-nitride quantum dots devices for solidstate lighting and solar cells, and novel approaches to reduce efficiency-droop issues in InGaN-based light-emitting diodes. She has authored or coauthored more than 15 refereed journal and conference publications.

Ms. Liu was the recipient of the Dean's Scholarship from Lehigh University in 2008 and the Best Graduation Thesis from the Huazhong University of Science and Technology in 2008.

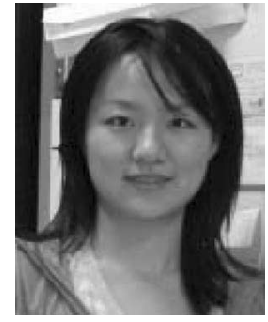

Jing Zhang received the B.S. degree in electronic science and technology from the Huazhong University of Science and Technology, Wuhan, China, in 2009. Since August 2009, she has been working toward the Ph.D. degree in the Department of Electrical and Computer Engineering and the Center for Optical Technologies, Lehigh University, Bethlehem, PA.

Her current research interests include III-nitride semiconductor nanostructures for thermoelectric and solid-state lighting technologies, various aspects of computational, growths, and device fabrication of III-nitride semiconductor for photonics and thermoelectric applications, and pursuit of novel materials for high thermoelectric figure of merit and solid-state lighting applications.

Ms. Zhang was the recipient of the Dean's Scholarship from Lehigh University in 2009 and the Best Graduation Thesis from the Huazhong University of Science and Technology in 2009.

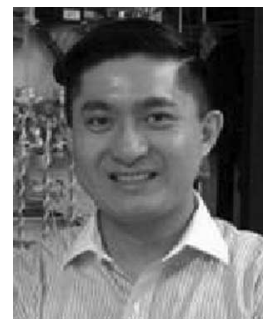

Nelson Tansu (S'99-M'02-SM'10) was born in October 1977. He received the B.S. degree in applied mathematics, electrical engineering, and physics (with highest distinction) and the Ph.D. degree in electrical engineering from the University of Wisconsin-Madison, Madison, in 1998 and 2003, respectively.

Since May 2009, he has been an Associate Professor (with tenure) in the Department of Electrical and Computer Engineering (ECE) and Center for Optical Technologies (COT), Lehigh University, Bethlehem, PA, where he was an Assistant Professor from 2003 to April 2007 and the Peter C. Rossin Assistant Professor from April 2007 to April 2009. He was the WARF Graduate University Fellow and Vilas Graduate University Fellow during his graduate studies at the University of Wisconsin-Madison. His current research interests include the theoretical and experimental aspects of the physics of semiconductor optoelectronics materials and devices, the physics of low-dimensional semiconductor (nanostructure), and metal-organic chemical vapor deposition, and device fabrications of III-nitride and III-V-nitride semiconductor optoelectronics devices on GaAs, InP, and GaN substrates. He has authored or coauthored in more than 180 refereed international journal and conference publications. He holds several U.S. patents (less than eight). He has been an Assistant/Associate Editor for the journal Nanoscale Research Letters since 2007. He was a Reviewer of the leading journals in applied physics, quantum electronics, nanotechnology, photonics, and optoelectronics areas. He has also given numerous lectures, seminars, and invited talks (over 40) in universities, research institutions, and conferences in the U.S., Canada, Europe, and Asia.

Dr. Tansu was in the Technical Program Committee for several major technical conferences for the IEEE, the Optical Society of America (OSA), the SPIE, and the American Physical Society (APS), which include IEEE/OSA Conference on Lasers and Electro-Optics (2007, 2008, and 2009), SPIE Photonics West (2009 and 2010), APS March Annual Meeting (2007, 2009, and 2010), and others. He was also an Invited General Participant at the 2008 National Academy of Engineering (NAE)'s U.S. Frontiers of Engineering (FOE) Symposium. He was in the Organizing Committee for the 2009 NAE's U.S. FOE. He was a Panel Member for U.S. National Science Foundation (Electrical, Communications and Cyber Systems, Division of Materials Research, Science and Technology Center Panel, and European Research Council Panel/Site Reviewer), U.S. Department of Defense, U.S. Department of Energy, several other U.S. federal agencies, private foundations in the U.S., and several funding agencies in Europe and Asia He was the Primary Guest Editor of the IEEE JOURNAL OF SELECTED TOPICS IN QUANTUM ELECTRONICS (2008-2009) and has been an Associate Editor of the IEEE PHOTONICS JOURNAL since 2009. He was the recipient of the Graduate Dissertator Award, the Harold A. Peterson Best ECE Dissertation Award, and the 2010 Wisconsin Forward Under 40 for Outstanding Young Alumni Award at the University of Wisconsin-Madison, and the Peter C. Rossin Professorship and the 2008 Libsch Early Career Research Award at Lehigh University. 